\title{
Augmented Reality, Cyber-Physical Systems and Robotic Surgery: Nice to Have or a Program with Future?
}

\author{
Chair: \\ Andreas Kirschniak ${ }^{\mathrm{a}}$ \\ Participants: Jan-Hendrik Egberts ${ }^{b}$ Frank-Alexander Granderath ${ }^{c}$ Sörgen Torge Mees ${ }^{d}$ \\ Markus Mille ${ }^{e}$ f Beat P. Müllerg $^{g}$ Tero Rautio $^{\text {h }}$ Anjali A. Röth ${ }^{f, i}$ \\ a Department of General, Visceral and Transplantation Surgery, University Hospital of Tübingen, Tübingen, Germany; \\ b Department of General, Visceral, Thoracic, Transplantation and Pediatric Surgery, University Hospital Schleswig Holstein, Campus Kiel, \\ Kiel, Germany; \\ 'Clinic of General and Visceral Surgery, Hospital Neuwerk 'Maria von den Aposteln', Mönchengladbach, Germany; \\ d Department of Visceral, Thorax and Vascular Surgery, Carl Gustav Carus University Hospital Dresden, Dresden, Germany; \\ e Department of General and Visceral Surgery, HELIOS Hospital Erfurt, Erfurt, Germany; \\ f Surgical Working Group of Young Surgeons (CAJC; chair and co-chair) of the DGAV (German Society for General and Visceral Surgery), \\ Berlin, Germany; \\ g Department of General, Visceral and Transplantation Surgery, University of Heidelberg, Heidelberg, Germany;

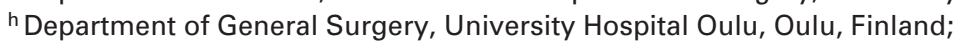 \\ i Department of General, Visceral and Transplantation Surgery, University Hospital RWTH Aachen, Aachen, Germany
}

\begin{abstract}
Question 1: The education in surgery has changed due to the introduction of laparoscopic techniques. Training can be performed by participating in courses or using simulators. Recently, the increasing popularity of robotic surgery has led to the spread of robotic systems, which allow new teaching strategies, e.g. by the use of a second console or telesurgery. In contrast, the majority of these procedures is carried out by senior surgeons, and less operations are performed as teaching operations. How will surgical education change due to the distribution of robotic surgery in your opinion?
\end{abstract}

Egberts: I think that teaching was never as good as with robotic systems, due to the following reasons:

(1) Simulator: It is an excellent training method with objective feedback (not like 'See one, do one, teach one' but with objective data about the training effect and success and transparent evidence about the training). The newer techniques allow the simulation of complete surgical procedures like lobectomy or low anterior rectum resections [1].

(2) Second console: The second console allows an excellent training and teaching facility due to the exact same perspective on the situs of mentor and mentee, and allows the possibility of guiding through the whole procedure step by step. When starting with a robotic system, senior surgeons operate in the begin- ning. In our hospital, we have been using the system since 4 years, and the robotic system is now integrated into the education of the whole team. Residence doctors are able to perform parts of the procedure on the second console. This allows new training concepts, and whole procedures can be taught in a modular way. If one briefs each other and defines the training parts before starting and when the points in time are clear when the operation is handed over again to the senior, a transparent concept of teaching is reached [2].

However, I also think that we need more time; currently we are at the stage where senior surgeons have to learn the technique. I think it will take several years for many hospitals to implement robotic surgery for the whole team.

Granderath: Traditionally, surgical expertise in conventional as well as in laparoscopic surgical procedures has been acquired in the operation room from experienced surgical proctors. In robotic surgery, to guarantee optimal patient care, surgeons have to be adequately trained before clinical use. Curricula, training courses, and fellowship programs have to be developed for maintaining definite requirements to obtain robotic operations. Proficiency in basic laparoscopy as well as technical certification has to be present. After proof of basic preclinical training, the clinical proficiency status must be obtained by an approved independent robotic surgeon.

Mees: The implementation of a well-structured training program is crucial for departments performing robotic surgery. In my

\section{KARGER}

() 2018 S. Karger GmbH, Freiburg
PD Dr. Andreas Kirschniak, MBA Department of General, Visceral and Transplantation Surgery University Hospital of Tübingen

Hoppe-Seyler-Straße 3, 72072 Tübingen, Germany andreas.kirschniak@uni-tuebingen.de 
opinion, the formation of teams training and operating together is mandatory to allow junior surgeons to observe and assist robotic operations in a frequent fashion before performing their first operations. Junior surgeons should start performing steps of the operations while being supervised by a senior robotic surgeon at a second console to take over the surgery in difficult situations to guarantee the safety of the patients.

Müller: One of the main benefits of robotic surgery is that it helps to shorten the learning curve for minimally invasive surgery. This will lead to a wider distribution of minimally invasive surgery even for more complex procedures, such as low anterior resections, esophagectomies, and pancreas resections. Novices will start at the console in a simulation environment and later can be supported by the senior surgeon from the teaching console. Finally, they will be able to do the surgery on their own much sooner when compared to conventional minimally invasive surgery. This might also be due to the fact that training conditions in the robotic field are more comparable to the intraoperative situation as compared to box trainers and pelvitrainers for conventional minimally invasive surgery.

Rautio: Until now, robotic surgery training programs have been available mainly for more experienced surgeons, and most of them have just passed their learning curve. At the moment we have to create new pathways for residents to learn robotic surgery. As already mentioned, along with the traditional simulator and cadaver courses, robotic platforms offer new ways for training besides proctoring arrangements in teaching hospitals.

Röth/Mille: In the future, more and more surgeries are going to be performed minimally invasively, and, therefore, laparoscopic surgery plays a very important role in surgical residency and education. Hence, it should be regularly included in all residency programs, which is currently not the case, e.g. in Germany. On the way to learning robotic surgery, it is essential that the resident or fellow learns conventional laparoscopic surgery first. In addition, one needs to be able to perform open surgery as well, as it is important for managing complications. With the aforementioned changes, the main parts of surgical residency will therefore take place at simulators in the future. For example, dual console systems could be used to train young surgeons in robotic surgery. Thereby, the teaching surgeon can easily hand over control over the instruments to the resident at any time. In a web-based survey conducted on general surgery residents in the USA in 2013, 64\% of all residents believed that formal training in robotic surgery is important in residency training [3]. We do agree but think that this should be limited to an overview in robotic surgery during residency so that robotic surgery remains a subspecialization.

\section{Question 2: The evidence of robotic surgery is a major point of discussion. The advantages in} comparison to laparoscopic surgery are not as clear as when comparing laparoscopic and conventional surgery. Which hypotheses should be answered in the near future to substantiate the advantages of robotic surgery? What are your major arguments in favor of and against robotic surgery? Does evidence in this field play an important role for you?

Egberts: As for every new technology, one asks for evidence and benefit in comparison to the existing techniques. However, just like with other techniques, time is needed to show the benefit of innovation (e.g. discussion about oncological laparoscopic rectum surgery). At the moment, the focus in the literature about robotic surgery is on the technical innovation itself, and in time, which is needed to prove its advantages to the existing strategies, new technologies will be offered, and the technological development cannot be stopped.

Robotic surgery is in the beginning of its evolution; in the future we will have integrated systems with augmented reality and integration of preoperative data such as computed tomography (CT) and magnetic resonance imaging (MRI). Intelligent systems could be possible and might be able to indicate mistakes or dangerous situations. Telemedicine will be a topic, too, so experts could be included into the operation scenario at any time. On the other hand, data management and privacy protection will be major topics in the future, too.

Because of rising costs for robotic systems centralization is bound to happen. In contrast, the costs for robotic surgery are accurate if one compares them to modern drug therapy or other diagnostic or therapeutic procedures. A surgical procedure should only be performed once - but in a perfect way.

Granderath: The main goal of all surgical innovations should be the patients' benefit. The specified advantages of robotic surgical procedures are supposed to be nearly the same as those of laparoscopic surgery versus conventional surgery. Due to smaller incisions, less operative trauma, and higher precision, robotic surgery has been reported to provide comparable benefits as for laparoscopic/open procedures, including shorter hospital stay, less blood loss, less pain, fewer complications, and faster return to normal activities. This has to be proven carefully in the future, and evidence in this field becomes necessary.

Mees: From my point of view, robotic surgery has clear advantages, such as enhanced suturing capability (e.g. esophagogastrostomy after esophagectomy) or improved visualization and improved articulation of instruments (e.g. ultra-low rectal resection).

Evidence-based medicine (EBM) plays an important role and has/will have a relevant impact on the reimbursement of robotic surgery in Germany. Nevertheless, there exists a lack of EBM for the majority of robotic surgeries. For example, the ROLARR study concluded that robot-assisted rectal resections did not reveal significant benefits compared to conventional laparoscopic surgery when performed by surgeons with varying robotic experience. The point is that this randomized controlled trial (RCT) mentions the 
varying expertise of surgeons. This indicates that the comparison of laparoscopic and robotic rectal resections might not have been adequate due to different capabilities of the participating surgeons. In summary, evidence is lacking and additional RCTs are needed.

Müller: The major arguments in favor of robotic surgery are the shortening of the learning curve, improvement of training conditions, better ergonomic conditions, and, most of all, enabling the performance of more complex procedures (such as esophagectomies and pancreas resections) in a minimally invasive approach. All of these benefits have not been investigated so far. The common observational and randomized controlled trials do not focus on such issues but rather on general clinical benefits in experienced hands at the end of the learning curve. However, it might be a fact that, overall, surgeons who complete their learning curve earlier produce fewer complications than others. Therefore, future research should focus on the learning curve and compare the clinical outcome of conventionally performed minimally invasive surgery and robot-assisted surgery by novices. Furthermore, it should focus on subgroups like obese male patients in lower rectal surgery and most complex minimally invasive procedures. The major drawback of robotic surgery is its high cost but it may be worthwhile in the long run by reducing the occurrence of complications.

Rautio: We have to remember that robotic surgery is nothing else but laparoscopic surgery. All the available data is based on the studies which have been done during the learning curve of robotic surgery. There are no studies about the surgeon's ergonomic issues, which should be paid attention to in future trials.

Röth/Mille: There are quite a few hypotheses that need to be answered to underline the advantages of robotic surgery. In our opinion, this is e.g. the ability to spare nerves during low anterior resections of the rectum, leading to fewer side effects such as erectile dysfunction. This might also be done with three-dimensional (3D) laparoscopy, but especially in the pelvis, the advantage of more degrees of freedom plays a crucial role. Evidence obviously is of high importance when considering the acquirement of a robotic system. On the other hand, indications for robotic surgery need to be explored continuously to achieve progress.

\section{Question 3: The costs for robotically assisted procedures are estimated to be EUR 2,000-3,000 Euro higher than for laparoscopic procedures. How does the aspect of higher costs affect you in the decision making in favor of or against the use of robotic systems? Do we have to consider cost aspects when introducing new technologies? Do you think that the introduction of new robotic systems can reduce costs and make robotic surgery more effective?}

Egberts: Counter question: Who mentioned the added costs of EUR 2,000-3,000? One has to differ between costs for the materials and those for the investment of the robotic system (the investment costs could be written off over many years, the costs per operation decrease when raising the number of procedures). On the other hand, some instruments could be spared in comparison to laparoscopic procedures. For a rectum resection, we have additional material costs of approximately EUR 600, not EUR 2,000-3,000. Having a look at personnel costs, it should be possible in the future that a trained operation nurse could handle the assistant job, if desired. Furthermore, new systems will help regarding the reduction of costs for robotic procedures.

Granderath: In Germany, the use of robotic systems in clinical practice is complicated by the dual reimbursement system. The cost of a robotic operation consists of direct and indirect costs. The main direct cost is buying the system, supplemented by a maintenance contract and costs for accessories and instruments (insufflator, HF devices, trocars, clips, etc.). Indirect costs arise through staff training (surgeon, assistants, etc.) and prolonged operation duration especially during the learning curve for these procedures. Therefore, the case-related costs amount to more than EUR 2,000 per operation. Taken together, these factors mean for a German clinic that the case cost per operation is usually the same for open, laparoscopic, or robotic surgery based on the Diagnosis-Related Group (DRG) system, which will result in a revenue deficit for the clinic. For the future, it has to be considered whether these costs decrease with an increase of cases; however, in the near future no cost-neutral operation will be possible for the individual hospital.

Mees: Economic aspects play an important role in our healthcare system. The importance of costs will increase in the future due to the demographic changes in our society. On the one hand, patients should be treated in the best possible way. On the other hand, the medical treatment needs to be affordable for our society. Thus, EBM is required to clarify which treatments are justified and indicated.

Müller: As a matter of course, a wider use of robotic systems and the implementation of other providers with new robotic systems on the market will lower the cost in the future. Apart from that, however, we should be open-minded about new technologies independently of the costs, at least until it is proven that they do not provide added benefit to the patients. Registries and well-designed controlled trials focusing on robotic-specific outcomes should absolutely accompany the implementation of robotic surgery in daily clinical practice. In the end, it will be a political decision whether robotic surgery should be covered by health insurance, but this is not a medical issue.

Rautio: The most important drawback of the robotic surgery is definitely high costs compared to laparoscopic surgery. So far it has been very hard to convince the hospital administration of the cost efficiency of robotic surgery. That is why we have been following our results and doing research work to find any advantages of robotics in comparison to traditional surgery. I hope that the upcoming new robotic platforms will crush the monopoly and cause a price war. 
Röth/Mille: As most clinics need to work economically, higher costs affect the decision of using robotic systems or not. This is particularly important in 'smaller' procedures and not so much the case in more complex visceral surgery such as Whipple procedures. On the other hand, costs should not be considered if there is evidence in favor of robotic systems, e.g. regarding nerve-preserving surgery. The introduction of new robotic systems will definitively lead to a cost reduction in our opinion. Nevertheless, more competition is necessary as it leads to better products and a higher efficiency.

\section{Question 4: Robotic techniques allow precise preparation and intuitive movements in the situs, and in many scenarios this can be much more easily achieved than by laparoscopic preparation. Which indications could possibly become technically feasible with the help of the robot? Do you think that new procedures will be feasible by the use of robotic systems only?}

Egberts: I think that many advanced laparoscopic procedures can be performed with the help of robotic systems, such as gastrectomies, esophagectomies, pancreatic resections, and complex rectum resections. With robotic systems the rate of minimally invasive procedures could rise. Currently, e.g., only $30 \%$ of rectal cancer operations in Germany are performed laparoscopically. This is comparable to many other entities, especially in oncological surgery.

Granderath: Optimal visualization and 3D imaging of the operative field as well as mechanical advantages over traditional laparoscopic surgery might be feasible across multiple surgical specialties. Currently, robotic surgery is used in urology, gynecology, and visceral/thoracic surgery and will certainly increase in pediatric and head/neck surgery. In my estimation, one substantial benefit of robotic surgery in the future will be performing operations with challenging access or limited operation areas.

Mees: Laparoscopy has improved dramatically within the last decades. Nowadays, a large variety of complex procedures can be performed by laparoscopic surgery. However, there are limitations that might be overcome by robotic surgery. For example, singleport surgery using the robot seems to be a promising option. For esophagectomies, a single-port approach via the neck could potentially allow an oncological dissection within the chest/mediastinum. In combination with a transhiatal approach, operating in the chest/access through the chest wall could be avoided.

Müller: At the very least, future surgical procedures will be performed by a minimally invasive approach more frequently and on a wider spectrum of indications than today. Prostatectomy is the most prominent example so far. Before the implementation of robotics into urology, prostatectomies were commonly done by open surgery because a minimally invasive approach was too complex for most urologists. Today it is the other way around. The same could be true for colorectal surgery, which is not performed in a minimally invasive fashion as widely as one would expect in Germany. However, for sure this will be true for more complex procedures like pancreas head resections and esophagectomies as well as for new minimally invasive procedures like sublay incisional hernia repairs.

Rautio: Even now robotic surgeons know how much easier it is to perform more complex operations like rectal cancer or hernia surgery by using the robotic system. I think that any laparoscopic procedure is technically feasible with the robot but some of the cases need to be done with traditional techniques.

Röth/Mille: In the last decades, more and more complex surgeries were performed minimally invasively by using conventional laparoscopic techniques. Hence, esophagectomies, pancreatic resections, or liver resections are increasingly being performed minimally invasively in selected patients today. The use of robotic systems will expand and refine this spectrum even more.

Generally, it should be noted that the use of robotic systems is justified in more complex surgeries, where space is limited or conventional laparoscopy has its technical limitations (e.g. limitation of degrees of freedom). In our opinion, the use of robotic systems will be particularly crucial in complex oncologic surgeries as lymphadenectomy is much more precise with a robotic system compared to conventional laparoscopy. SILS (single-incision laparoscopic surgery) and NOTES (natural orifice transluminal endoscopic surgery) will benefit from robotic systems due to more degrees of freedom and better triangulation. Therefore, single-site surgery will be even better applicable, especially in more complex interventions.

\section{Question 5: Which tools should be integrated in a robotic system? Which devices and which connections to other data could you imagine?}

Egberts: I would appreciate to integrate preoperative data such as MRI and CT, more stapler devices, and a connection to the hospital data management systems.

Granderath: One interesting tool in a robotic system could be the integration of preoperative patient data (CT, MRI, etc.) in the system. Certainly, anatomical or physiological information can be connected with the robotic interface and improve the surgical procedure. Additionally, smaller instruments for microsurgical procedures could be useful.

Mees: As already mentioned, single-port surgery could be an interesting aspect in robotic surgery. In addition, devices for the dissection of liver parenchyma (e.g. ultrasound aspiration technique) would be very helpful to optimize robotic liver surgery.

Thinking about cognition-guided surgery, I could imagine that scans/images will be transferred into the field of view of the surgeon, demonstrating anatomical landmarks or pathologies to help the surgeon in performing his surgery. 
Müller: Firstly, robotic systems should become more streamlined so that they can be better integrated in the operating room workflow. One of the most important tools in this regard would be the connection to the operation table so that it can be moved jointly with the robot. Secondly, imaging tools and navigation would be nice features to have in the future. A robot as an electromechanical and computer-controlled device with its own imaging technology and mechanical tracking opportunity would be predestined for this. Such tools could help in opening the surgical field for wider robot applications e.g. in liver surgery.

Rautio: It would be nice to have tools for the assessment of surgical technical skills like there are already available in simulator systems. Navigating CT scan/MRI-based systems will be available in the near future.

Röth/Mille: A major advantage could be the combination of robotic systems and real-time image guidance based upon CT/MRI scans, which are fused with the $3 \mathrm{D}$ image of the console. In addition, functional data may be included such as liver capacity and, thus, the predictive liver function after resection. This may result in a detailed preparation technique and in performing more radical procedures. Also, eye tracking could be integrated to allow navigation in the surgical field and easy instrument selection.

A major improvement would also be haptic feedback which allows the surgeon to not only see but also feel the structure of the tissues in the situs.

\section{Question 6: Currently, the robot is a remote- controlled system. Neglecting legal aspects, could you imagine the robot to perform surgical steps automatically (e.g. trocar placement, suturing)? Which steps would you allow to be performed automatically, presupposing that the robot would be safe and as precise as a human?}

Egberts: I am not sure if automatic operation such as suturing an anastomosis will come soon. For sure, telemedicine will become more important.

Granderath: I am not sure whether we cross the ethical border at this point. Technically, I am quite convinced that computers/robots will be able to perform single steps in the future without a surgeon.

Mees: I have significant concerns letting the machine act independently. Nonetheless, I could imagine that the robot could resect liver tumors when preoperative scans are integrated into the robotic systems, allowing the device to precisely identify anatomical structures. In summary, the patient's safety is most important, and at this point in time I do not see the robotic system perform anything without human control.

Müller: It cannot be assumed that robots will ever act completely autonomously in the field of soft tissue surgery like visceral surgery. However, subtasks might possibly be conducted automatically in the future, e.g. the suturing of an anastomosis, resection of parts of parenchymatous organs, or tubular resection of visceral organs. However, trocar placement would not be a good example because the robot first needs to be connected and registered to the patient before it can perform an automatic task. Furthermore, in my opinion, automatic robot trocar placement seems to be too high of a risk in terms of injury.

Rautio: At the moment I cannot imagine automatically performed steps of the surgical procedure. Instead there will be robotic navigating systems for abdominal biopsies.

Röth/Mille: In the literature, there are some papers that explore this approach. The major drawback always seems to be technical difficulties, which makes it difficult to predict the applicability. Nonetheless, the surgeon always needs to play a supervising role, being able to intervene at any time (comparable to driver assistance systems in automotive industry). On the other hand, the robot might play an important role as a supervisor for the surgeon, e.g. with an alarm signal whenever blood vessels such as the portal vein are within the preparation plane.

\section{Question 7: Robotic systems might extend today's} limitations of telemetric medicine. How do you judge its role in supraregional device sharing and telesurgical consultation of specialists, e.g. in rural areas?

Granderath: Telemedicine/telesurgery can offer significant possibilities in the future. Based on functioning networking and clarification of legality, surgical procedures as well as training sessions can be performed by experienced surgeons/experts in other hospitals /training centers without personal presence.

Mees: At the moment, I do not see a role for robotic surgery in a telemetric fashion. Nowadays, patients can be transferred to specialized hospitals within a reasonable time frame. The problem with telemetric robotic surgery is that a specialized surgeon/assistant needs to be in the rural hospital to assist with the robot and/or handle robotic complications. Thus, robotic procedures should not be performed in small rural hospitals without specialized staff on site. This well-trained staff is certainly not available in the countryside; therefore, not the staff should travel but the patient should be transferred to a specialized unit.

Müller: In 2001, it was demonstrated by Jacques Marescaux that telesurgery is possible when he operated on a patient in Strasbourg while he himself was located in New York. However, doing so they also recognized the huge safety and medicolegal problems associated with this technology. It has to be noted that since then no broader use of telesurgery has been established. Therefore, it remains unclear whether telesurgery in a broader use will be implemented in the future. Prior to this, most larger clinics will likely have their own robotic systems, und smaller clinics will refer their complex patients to those. 
Rautio: Robotic surgery systems will empower eternal possibilities for telesurgical consultation or even 'hands-on' assistance in case of a difficult operation.

Röth/Mille: There are a lot of challenges in telementoring and telesurgery. Hung et al. [4] have addressed these challenges and categorized them into safety, legal, financial, and ethical challenges. Additionally, there are also technical challenges, e.g. internet connection or IT premises. Even if these challenges are met, a visceral surgeon who is experienced in managing the possible complications is necessary. Besides these concerns, we see telesurgical consultation of specialists in rural areas also very critically as an operative procedure that is performed on a patient is not only a matter of what takes place in the operating room but also perioperative management (intensive care unit, radiological intervention) of the patient plays an important role. There is a lot of evidence that proves that morbidity and mortality decreases significantly if complex surgery is performed at a center with a high case volume in this specific entity. This is not only due to surgical experience alone but also because of perioperative management.

\section{Participants}

Prof. Dr. Jan-Hendrik Egberts

Klinik für Allgemeine, Viszeral-, Thorax-, Transplantations- und

Kinderchirurgie

Universitätsklinikum Schleswig Holstein, Campus Kiel

Arnold-Heller-Straße, 24105 Kiel, Germany

Jan-Hendrik.Egberts@uksh.de
Prof. Dr. med. Frank A. Granderath

Allgemein- und Viszeralchirurgie

Krankenhaus Neuwerk "Maria von den Aposteln"

Dünner Straße 214-216, 41066 Mönchengladbach, Germany

chirurgie@kh-neuwerk.de

Prof. Dr. med. Sören Torge Mees

Klinik und Poliklinik für Viszeral-, Thorax- und Gefäßchirurgie

Universitätsklinikum Carl Gustav Carus

Fetscherstraße 74, 01307 Dresden, Germany

soeren.mees@uniklinikum-dresden.de

Dr. med. Dr. med. univ. Markus Mille

Allgemein- und Viszeralchirurgie

HELIOS Klinikum Erfurt

Nordhäuser Straße 74, 99089 Erfurt, Germany

markus.mille@ helios-gesundheit.de

Prof. Dr. med. Beat P. Müller

Klinik für Allgemein-, Viszeral- und Transplantationschirurgie Universitätsklinikum Heidelberg

Im Neuenheimer Feld 110, 69120 Heidelberg, Germany

BeatPeter.Mueller@med.uni-heidelberg.de

Tero Rautio, MD, $\mathrm{PhD}$

Department of General Surgery

University Hospital Oulu

Oulu, Finland

tero.rautio@ppshp.fi

Dr. med. Dipl. Phys. Anjali A. Röth

Klinik für Allgemein-, Viszeral- und Transplantationschirurgie Uniklinik RWTH Aachen

Pauwelsstraße 30, 52074 Aachen, Germany

aroeth@ukaachen.de

\section{References}

1 Cerfolio RJ, Cichos KH, Wei B, Minnich DJ: Robotic lobectomy can be taught while maintaining quality patient outcomes. J Thorac Cardiovasc Surg 2016;152: 991-997.
2 Tou S, Bergamaschi R, Heald RJ, Parvaiz A: Structured training in robotic colorectal surgery. Colorectal Dis 2015; 17:185.

3 Farivar BS, Flannagan M, Keitman IM: General surgery residents' perception of robot-assisted procedures during surgical training. J Surg Educ 2015;72:235-242.
4 Hung AJ, Chen J, Shah A, Gill IS: Telementoring and telesurgery for minimally invasive surgery. J Urol 2018; 199:355-369. 